\title{
Sound propagation in sodium di-2-ethyl-hexylsulfosuccinate micelles and microemulsions
}

\author{
L. Ye, D. A. Weitz, Ping Sheng, and J. S. Huang \\ Exxon Research and Engineering Company, Route 22 East, Annandale, New Jersey, 08801
}

(Received 17 May 1991)

\begin{abstract}
We present the results of a systematic study of the propagation of sound in sodium di-2-ethylhexylsulfosuccinate (AOT) micelles and microemulsions. The dispersion in the sound velocity $v$ is determined over three and a half decades in frequency by using both ultrasonic and Brillouin-scattering techniques. The dispersion in the sound velocity is also measured as a function of the volume fraction $\phi$ of micelles or microemulsions. In addition, we measure the dependence of the sound velocity dispersion on the linear hydrocarbon chain length of the solvent molecules, and on the size of the microemulsion droplets. A consistent physical picture emerges that accounts for all of the results. The sound velocity in the micelle or microemulsion phases is greater than that in the solvent, leading to the observed increase of $v$ with $\phi$. In addition, due to the overlapping of the surfactant tails, there is a weak, short-range attractive interaction between the droplets, causing them to form short-lived, extended networks. These networks can support shear, leading to a further increase in $v$ at higher $\phi$, provided the frequency of the sound is sufficiently high that the instantaneous networks remain intact over the period of the sound wave. This results in the additional frequency dispersion in $v$ at high $\phi$. The strength of the attractive interaction, and hence the dispersion in the sound velocity, depends on the chain length of the solvent molecule and the diameter of the microemulsion droplet. The use of an effective-medium model is critical in confirming the validity of the physical picture. The effective-medium model includes the contribution of a shear modulus of one of the phases and can account for the $\phi$ dependence of $v$ for all the systems. The shape of the full Rayleigh-Brillouin spectra is shown to be describable by a formalism that includes the relaxation of the extended networks. Finally, since the micelle or microemulsion networks cannot support shear unless they extend across the whole system, we show that the additional shear modulus contributed by the droplet phase exhibits scaling behavior when the volume fraction exceeds a critical value defined by the rigidity percolation threshold. This allows us to measure both the critical volume fraction and the exponent for rigidity percolation. However, since this additional shear modulus only occurs at high frequency, this effect is an example of dynamic rigidity percolation.
\end{abstract}

PACS number(s): 62.30. $+\mathrm{d}, 43.35 .+\mathrm{d}, 62.80 .+\mathrm{f}$

\section{INTRODUCTION}

The propagation of sound in a fluid can provide a sensitive probe of its physical properties [1-5]. At low frequencies, the sound velocity provides a measure of the compressibility, and hence the elastic modulus, of the fluid, while the sound attenuation provides a measure of the viscosity of the fluid [3-5]. At higher frequencies, fluids can become viscoelastic, and measurement of the sound propagation can provide a probe of the elastic solidlike properties of the fluid $[1,2]$. As the frequency of the sound increases above the relaxation frequency of the internal motions within the fluid, these processes can no longer respond to the sound wave, and are "frozen in," resulting in an increase in the elastic modulus of the fluid, and a concomitant increase in the sound velocity. Thus the frequency dispersion of the sound velocity provides a direct measure of the additional relaxation processes that contribute to the viscoelastic behavior of the fluid.

Sound propagation in fluids can be experimentally measured using ultrasonic techniques, which can probe the frequency range of $\sim 1-50 \mathrm{MHz}$. Higher-frequency measurements can be made using Brillouin scattering, which probes the frequency range from $\sim 0.5$ to $10 \mathrm{GHz}$. Both methods have been extensively applied to study sound propagation in simple fluids, in molecular liquids, and in polymer melts. However, considerably less attention has been devoted to the study of sound propagation in complex fluids, which possess structures on length scales larger than the molecules that comprise the fluids. These larger scale structures will possess additional relaxation mechanisms, which can be reflected in the frequency dispersion of the sound propagation. Thus the study of sound propagation in complex fluids can provide new insight into these structures and their dynamics.

In this paper, we present a comprehensive study of the sound propagation in one complex fluid system, providing an example of the richness of the physical properties that can be observed. We study a system of inverted micelles [6] and microemulsions [7] formed with the surfactant, sodium di-2-ethyl-hexylsulfoscuccinate (AOT), water and oil. This is a widely studied system whose phase behavior and structure is well known. In this system, oil is the continuous phase. In the absence of water, the surfactant molecules aggregate together to form spherical inverted micelles, with the head groups forming a more rigid central core, and the hydrocarbon chains sticking out into the solvent oil $[8,9]$. Each micelle is comprised of 22 molecules, and has a radius of $15 \AA$. As more surfactant is added, the volume fraction of micelles in the solution $\phi$ 
increases, but both the size and the shape remain unchanged, allowing us to measure the propagation of sound as a function of $\phi$. Upon addition of water, a microemulsion is formed as the micelles are swollen to form spherical droplets with water in the central core, surrounded by a layer of surfactant molecules, which again have their head groups facing the water and their tail groups facing into the solvent oil. The ratio of water to surfactant determines the diameter of droplets, and, provided this ratio is held fixed, the volume fraction of droplets can again be varied without changing their shape or size. Both the micelle and the microemulsion droplets have a weak, short-range attractive interaction. This can result in the formation of transient structures with much larger length scales, which dramatically affect the propagation of sound. The study of sound propagation in this system provides information which helps elucidate both the nature of these structures and their dynamics.

We measure the sound velocity using both ultrasonic techniques and Brillouin scattering. The ultrasonic measurements are used to study the frequency range from 2 to $30 \mathrm{MHz}$, while the Brillouin-scattering measurements extend from 0.7 to $10 \mathrm{GHz}$. This allows us to measure the dispersion in the sound velocity over a wide range of frequency. For each system, we measure the velocity and attenuation of the sound propagation as a function of both frequency and droplet volume fraction. In addition, we vary the magnitude of the attractive interaction between the droplets by changing the chain length of the linear hydrocarbon of the solvent oil, and by increasing the diameter of the microemulsion droplets by adding additional water. The interpretation of our results depends critically on the use of an effective-medium model to describe the dependence of the sound velocity on $\phi$. Using this interpretation, we develop a self-consistent picture which accounts for all of our data and provides new insight into the interactions and dynamics of the droplets in the micelles and microemulsions.

The essential physics of our picture can be summarized by considering the behavior of micelles in decane. We find that the sound velocity $v$ increases significantly as the volume fraction of micelles is increased, indicating that the elastic modulus of the AOT is larger than that of the decane. However, as the frequency of sound is increased, there is an additional dispersion in the sound velocity at larger volume fractions, $\phi \geq 0.16$. The key to our interpretation of this behavior is the transient structures that are formed by the droplets due to the weak attractive interaction between them. Because of this interaction, the droplets can form extended networks, that will maintain their integrity for some characteristic time $\tau_{c}$ determined by the strength of the attractive interaction. When the frequency of the sound is greater than the corresponding characteristic frequency $f_{c}=1 / \tau_{c}$, the extended networks maintain their integrity for the full period of the sound wave, and have a profound influence on the propagation of sound. By contrast, when the frequency of the sound is less than $f_{c}$, the extended networks relax in a time less than the period of the sound and thus make no contribution to the sound propagation. The extended networks can support shear, and thus, at high frequen- cies, the additional contribution of this shear modulus results in an increase in the total elastic modulus of the system, and hence an increase in the sound velocity. The additional, frequency-dependent dispersion is only observed at higher volume fractions because the extended networks must span the full volume of the system in order to support shear. Thus the volume fraction of micelles must be above a critical value of $\phi_{c}=0.16$ in order to observe the increase in the sound velocity. Furthermore, the contribution of the additional shear modulus depends on the extent of the networks, and exhibits a remarkable scaling behavior, consistent with percolation of the micelles. From the data, we are able to experimentally determine the exponent $\tau$ for the elasticity modulus of a percolating system. We call this behavior dynamic rigidity percolation.

In this paper, we investigate the extent of the applicability of this physical picture as the system is modified. Thus, we first consider the effects of varying the solvent oil in the micelle system. We restrict ourselves to linear chain hydrocarbons, and vary the oil from hexane $\left(\mathrm{C}_{6} \mathrm{H}_{6}\right)$ to hexadecane $\left(\mathrm{C}_{16} \mathrm{H}_{16}\right)$. Increasing the chain length of the oil is known to increase the strength of the attractive interaction between the micelles [10]. We find that the effect of increasing the attractive interaction is to decrease $f_{c}$. We also investigate the effect of swelling the droplets with water to form microemulsions. This has two, competing effects. By increasing the radius of the droplets, and hence the area of their interaction, the attractive interaction between them is increased. Thus, for small microemulsions, with a low water content, an additional, frequency-dependent dispersion is again observed, with the onset frequency of the additional dispersion dependent on the chain length of the oil solvent. In addition, we again observe dynamic rigidity percolation, with the same values of both $f_{c}$ and $\tau$. However, the droplets are swollen by water, which has a lower elastic modulus than the AOT, and cannot, by itself, support shear. Thus, as the size of the droplet is increased still further, the intrinsic elastic modulus of the droplets themselves is decreased, and they can no longer support shear, so no additional, frequency-dependent dispersion is found. Thus our basic physical picture can describe the behavior of both the micelles and microemulsions for a variety of different solvent molecules.

The remainder of this paper is organized as follows. In the next section we discuss the micelles and microemulsions in greater detail. We then present a complete description of the two effective-medium models [11] that we employ, one to describe the behavior at very low frequencies when there is no contribution of a shear modulus of the droplet phase, and the second that includes this contribution. The following section provides the details of our experimental techniques. A complete discussion of the results follows, beginning with the description of the observations of the dependence of the frequency dispersion of $v$ on the chain length of the oil for the micelles followed by the dependence on the size of the microemulsions. We then compare the observation of dynamic rigidity percolation in micelles with that in microemulsions. Next, we discuss the details of the 
Brillouin-scattering spectra that are observed and use a model [12] that includes the consequences of internal relaxations to fit the spectra. The results obtained are in good accord with our direct observations of the frequency dependence of $v$. Finally, we also describe the observation of an additional dispersion in $v$ at very high frequencies which persists for all systems and which we attribute to the relaxation of the hydrocarbon tail groups of the AOT molecule itself. A brief summary concludes the paper.

\section{AOT MICELLES AND MICROEMULSIONS}

The surfactant molecule, sodium di-2-ethylhexyl sulfosuccinate (AOT), has a hydrophilic head and a hydrocarbon hydrophobic tail [13]. As a result, the surfactant molecules are preferentially adsorbed on the interface between water and oil, forming equilibrium structures that are larger than the molecular scale. The size and shape of these structures are determined by the competition between the minimization of the interfacial energy, the curvature energy due to the bending elasticity of the surfactant tail layer, and the entropy [10]. In the systems studied here, all of the structures are spherical droplets $[8,9]$, and the continuous phase is a simple, linear alkane: hexane $\left(\mathrm{C}_{6} \mathbf{H}_{14}\right)$, decane $\left(\mathrm{C}_{10} \mathrm{H}_{22}\right)$, or hexadecane $\left(\mathrm{C}_{16} \mathbf{H}_{34}\right)$. When there is no water in the mixture, the hydrophilic heads of the AOT molecules aggregate together to avoid the oil phase, and the resultant spherical structure contains roughly 22 AOT molecules with radius of $15 \AA$ $[8,13]$. Since the oil is the continuous phase, this structure is usually called an inverted micelle. When water is added to the system, the heads of the surfactant molecules associate with the water molecules and form droplets with water cores. As more water is added, the droplets are further swollen and their radius increases. The size of the droplets is determined by the molar ratio of water to surfactant, $R=\left[\mathrm{H}_{2} \mathrm{O}\right] /[\mathrm{AOT}][10]$. The number of AOT molecules in each droplet varies with the size of the droplet and the amount of water added, keeping the packing density of the AOT on the interface constant. For example, there are about 500 AOT molecules in each droplet when the radius is $50 \AA$ [14]. The sizes of microemulsions we have studied are 25,45 , and $75 \AA$ in radius, corresponding to $R$ of 8,25 , and 41 , respectively.

Both the micelle and the microemulsion systems are thermal equilibrium structures [10]. The fluctuations in droplet shape and size are small. A key feature of these systems, which we exploit in all of our measurements, is the fact that the droplet size is insensitive to the concentration of droplets. Therefore, by adding oil, we can dilute the droplet concentration, or volume fraction, $\phi$, without changing the size. However, since the micelles only form when the AOT concentration is above a critical value, $[\mathrm{AOT}]=0.225 \mathrm{mM}[14,15]$ we change $\phi$ by diluting with a $0.9-\mathrm{m} M$ solution of AOT in oil. This enables us to study the $\phi$ dependence of the sound velocity. For the micelles, we define $\phi$ as the volume fraction of micelles and calculate it directly from the volume fraction of surfactant added. For the microemulsions, we define $\phi$ as the volume fraction of water and surfactant added. Provided $R$ is maintained constant, the droplet size is independent of $\phi$. In both cases, there is some ambiguity about the meaning of the volume fraction. Although we treat the droplets as hard spheres with a welldefined radius, and hence a well-defined $\phi$, the droplets are in fact somewhat "fuzzy" as the solvent oil molecules can penetrate, to some extent, within the hydrocarbon tails of the AOT. In addition, the droplets are not rigid, but can deform. Thus, it is possible to obtain packing fractions that are higher than those possible with hard spheres. However, in this work we restrict our attention to $\phi<0.6$, and view the droplet as essentially hard spheres.

There is a weak, short-range, attractive interaction between the droplets $[10,15]$. Physically this is due to the overlapping of the short, branched, hydrophilic surfactant tails of droplets. The range of this overlap is about 3 $\AA$. The attractive interaction is an entropic effect. For the oil solvent molecules to fit in between surfactant tails, they must adopt certain configurations in their phase space, thereby decreasing their entropy. By contrast, the tails of the surfactant molecules in a neighboring droplet are already optimally oriented by the droplet structure and are able to interpenetrate without suffering as much decrease in their entropy. Thus, the total free energy of the system is lower when the surfactant tails of two droplets interpenetrate than when solvent oil molecules fit in between the surfactant tail groups of the droplets. This results in the net attractive interaction. The strength of this attractive interaction increases as the length of the solvent oil molecule increases, because the relative entropic cost for oil molecules to fit in between surfactant tails also increases [14]. In addition, for the microemulsions, as the droplet size increases, the area of overlapping surfactant tails also increases, leading to a larger interaction energy [8]. This interaction energy determines the characteristic time scale of the interaction $\tau_{c}$. Therefore, we can vary $\tau_{c}$ either by using different oils or by changing the droplet size.

\section{EFFECTIVE-MEDIUM THEORY}

Effective-medium theory is used to calculate the effective characteristics of an inhomogeneous system measured in the long-wavelength limit. The theory is derived from the consideration of an inclusion isolated in an infinite effective matrix, whose properties reflect the average effects of other inclusions $[11,16]$. Each of the constituent phases of the medium is described by parameters appropriate for the pure phase, and effective-medium theory is used to determine the parameters that describe the composite medium as a function of the relative volume of the constituent phases. Thus, effectivemedium theory can be used to evaluate the elastic modulii for multicomponent composites [16], and to calculate the $\phi$ dependence of the average elastic modulus $\beta$. In this paper we make extensive use of effective-medium theory to account for the elastic behavior of the micelle and microemulsion systems, since the relevant acoustic wavelength is always orders of magnitude larger than the size of the micelles and microemulsions. In our experi- 
ments, the wavelength of the sound is always greater than $2000 \AA$, while the size of droplets is always smaller than $200 \AA$. In fact, the sound wavelengths are usually much larger, while the droplet sizes are often much smaller. In our approach, we take one phase to be the continuum fluid, while the second phase consists of micelle or microemulsion inclusions randomly embedded in the continuous phase. We describe each of the constituent components, the oil phase and the droplet phase, in terms of five parameters. Thus the oil is described by the density $\rho_{0}$, the complex longitudinal-wave elastic modulus $\beta_{0}=\beta_{0}^{\prime}+i \beta_{0}^{\prime \prime}$, and the complex shear modulus $\mu_{0}=\mu_{0}^{\prime}+i \mu_{0}^{\prime \prime}$. The droplet phase is described by the same set of parameters with the subscripts replaced by $d$. We then use the effective-medium theory to determine the average value of complex elastic modulus, $\beta=\beta^{\prime}+i \beta^{\prime \prime}$ as a function of $\phi$. The measured sound velocity $v$ is then given by

$$
v=\sqrt{\beta^{\prime} / \rho},
$$

where $\rho$ is the average density, given by $\rho=\phi \rho_{d}+(1-\phi) \rho_{0}$. The attenuation of the sound is determined from $\beta^{\prime \prime}$. It is related to the measured ultrasonic attenuation $\alpha$ by $\beta^{\prime \prime}=\alpha 4 \pi v^{3} \rho / f$, and to the measured full width at half maximum (FWHM) of the Brillouin peak $\Delta \omega$ by $\beta^{\prime \prime}=\Delta \omega^{2} v^{2} \rho / f$. We can define a generalized attenuation of the sound velocity through

$$
\Delta v=\sqrt{\beta^{\prime \prime} / \rho} .
$$

The frequency dependence inherent in the viscoelastic behavior exhibited by our systems represents an additional complexity in the theory. An effective-medium model is a static theory, with no inherent frequency dependence apart from that which is present in the parameters of the constituent phases. Therefore, to account for our observations, we allow the parameters which describe each phase to vary with frequency, and determine their values from the $\phi$ dependence of the sound velocity at each frequency independently.

At high frequencies, a random network is formed by the surfactant phase, and we must account for the possibility that this network supports shear. Thus, we model the system with a symmetric model for the two phases, and the relevant effective-medium equations are [8]

$$
\begin{aligned}
& \frac{1}{\beta}=\frac{\phi}{\beta_{d}+4 / 3\left(\mu-\mu_{d}\right)}+\frac{1-\phi}{\beta_{0}+4 / 3\left(\mu-\mu_{0}\right)}, \\
& \frac{1}{\mu+H}=\frac{\phi}{\mu_{d}+H}+\frac{1-\phi}{\mu_{0}+H},
\end{aligned}
$$

where

$$
H=\mu \frac{9 \beta-4 \mu}{6 \beta+4 \mu} .
$$

It should be noted that these equation reflect the fact that the longitudinal elastic modulus is modified by the shear modulus, because the droplet phase of the system reacts elastically in response to an applied strain.

At low frequencies, the micelles do not support shear because any applied shear will be relaxed, since the net- works do not persist for a full period of the sound wave. We thus use a model which treats the micelles as independent droplets. The effective elastic modulus $\beta$ is calculated using Wood's approximation [17], which weights the inverses of $\beta_{0}$ and $\beta_{d}$ with their volume fractions,

$$
\frac{1}{\beta}=\frac{\phi}{\beta_{d}}+\frac{1-\phi}{\beta_{0}} .
$$

This simple equation may be deduced from Eq. (2) by setting $\mu_{0}=\mu_{d}=0$.

In order to calculate the effective complex elastic modulus $\beta$, the elastic properties for the two constituent phases must be known. In our systems, all the required parameters for the oil are known either from the literature values [18] or from measurements of the sound velocities, viscosities, and indices of refraction. The elastic modulus of the pure oil $\beta_{0}$, is determined from the measured sound velocity and the density $\beta_{0}^{\prime}=v_{0}^{2} \rho_{0}$. The imaginary part of the elastic modulus of the oil $\beta_{0}^{\prime \prime}$ is determined from the measured damping of sound in the pure oils, using either $\alpha$ or $\Delta \omega$. The low-frequency shear viscosity $\eta_{0}$, measured using a viscometer, is used to determine the imaginary shear modulus $\mu_{0}^{\prime \prime}=i \omega \eta_{0}$. Since a fluid cannot support shear, we set $\mu_{0}^{\prime}=0$.

A pure phase of AOT configured as droplets does not exist, so most of the parameters describing the surfactant phase cannot be determined experimentally. The only parameter that is known is the density, $\rho_{d}=1.13 \mathrm{~g} / \mathrm{cm}^{2}$. Thus, we have four unknown parameters describing the AOT phase which must be obtained from a fit to the data. These are the complex elastic modulus $\beta_{d}$ and the complex shear modulus $\mu_{d}$. At low frequencies, the relaxed droplet network cannot support shear, and we use Wood's approximation to calculate $\beta$, with $\mu_{d}=0$. However, at high frequency, where the extended network of droplets can support shear, we use both $\mu_{d}^{\prime}$ and $\mu_{d}^{\prime \prime}$ as fitting parameters.

The effective elastic modulus $\beta$ in Eq. (2) is calculated by recasting the equations into a polynomial equation in $\mu, 2 \mu^{4}+M_{3} \mu^{3}+M_{2} \mu^{2}+M_{1} \mu+M_{0}=0$, where $M_{i}$ are coefficients determined by $\phi, \beta_{0}$, and $\mu_{0}$ and the fitting parameters $\beta_{d}$ and $\mu_{d}$. The roots of the polynomial equation are determined, and the proper root is chosen by ensuring that both $\mu^{\prime}$ and $\mu^{\prime \prime}$ are positive. We then calculate $\beta$ from Eq. (2a), and finally determine $v$ and $\Delta v$.

We use a nonlinear least-squares routine to find the optimal values of the fitting parameters to describe the volume fraction dependence of the data, $v(\phi)$ and $\Delta v(\phi)$. Since the measurements of the sound velocity are much more reliable than those of the sound damping, we give a larger weight, by a factor of 5 , to $v(\phi)$ than to $\Delta v(\phi)$. We find that the fitting procedure is robust, and is relatively insensitive to the choice of initial values for the fitting parameters. Finally, since the effective-medium theory assumes that both components in the system are space filling, while hard spheres cannot fill space, we adjust $\phi$ by multiplying by 0.64 , corresponding to the volume fraction for random close packing of spheres [19]. 


\section{EXPERIMENT}

We use the AOT surfactant from Fluka without further purification, while the solvents, decane, hexane, and hexadecane, are all Gold label from Aldrich. The droplet volume fraction is determined from the composition of each component. Each material is added by weight and then converted to volume fraction using the bulk densities. Because there is some penetration of the oil into surfactant tails, the actual values of $\phi$ are slightly less well defined.

In the Brillouin-scattering experiments, the sound velocity is determined from the measurement of the frequency shift of the peak of the Brillouin doublet $\omega$ by $v=\omega / q$. Here the scattering wave vector is given by $q=(4 \pi n / \lambda) \sin (\theta / 2)$, where $\theta$ is the scattering angle, $n$ is the index of refraction of the system, and $\lambda$ is the wavelength of the light in vacuum. The scattering angle is determined approximately from the geometry of the optics, and then is determined more precisely through a calibration procedure using simple liquids with known sound velocities. The index of refraction is measured by a refractometer for each sample to an accuracy of \pm 0.001 . The measurement result of the $\phi$ dependence of $n$ for AOT micelles in decane is shown in Fig. 1 . We find that the experimental data are well described by $n(\phi)=(1-\phi) n_{0}+\phi n_{d}$, where $n_{0}=1.4113$ is the measured index of refraction for pure oil solvent, and $n_{d}=1.448$ is the fitting parameter, representing the phase consisting of AOT micelles. We also find that this value of $n_{d}$ can fit the results of $n(\phi)$ for all other samples.

Our Brillouin-scattering experiments are performed using either an $\mathrm{Ar}^{+}$laser at $5145 \AA$ or a $\mathrm{Kr}^{+}$laser at 6471 $\AA$. An etalon in the cavity ensures that the laser operates at a single longitudinal mode, providing a single frequency. The laser power we use is typically about $100 \mathrm{~mW}$ measured at the sample. The laser beam is focused by a 100 -mm-focal-length lens to a spot of about $100 \mu \mathrm{m}$ in diameter in the sample cell. Scattering angles between $15^{\circ}$ and $175^{\circ}$ are used, allowing us to vary the measured sound frequency $f$ from 0.7 to $9 \mathrm{GHz}$. The sample container is a $10 \times 10-\mathrm{mm}^{2}$ square glass cuvette. The scattered light is collected with a camera lens with focal

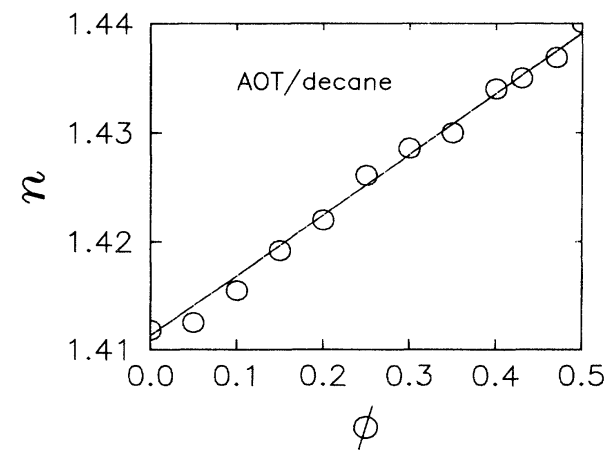

FIG. 1. Index of refraction of the AOT-decane micelle system as a function of drople volume fraction. The solid line is a fit using $n=(1-\phi) n_{0}+\phi n_{d}$ with $n_{d}=1.448$ and $n_{0}=1.4113$. length of $75 \mathrm{~mm}$. The excited volume is at the focal plane of the lens, ensuring that the collected light is collimated into the Fabry-Pérot device. The collimated beam then passes through a piezoelectrically driven, scanning, Fabry-Pérot interferometer with stabilization controls. The signal from the Fabry-Perot device is focused into a photomultiplier operating in the photoncounting mode. The data are collected with a multichannel analyzer.

The mirror set of the Fabry-Pérot device has a diameter of 2 in. and $88 \%$ reflectivity at the wavelengths used. All the measurements reported here are obtained with the Fabry-Pérot device operated in a five-pass configuration. This is achieved by using two corner cube retroreflectors on either side of the mirrors. These displace the transmitted beams laterally and pass them through the mirror set five times. The aperture of the five-pass operation is $7 \mathrm{~mm}$ in diameter. The five-pass operation offers high resolution and high contrast. The finesse in one pass is about 20, while in five-pass operation it is about 50. Thus the resolution is about $2 \%$ of the free spectral range $\left(\omega_{\mathrm{FSR}}\right)$ of the Fabry-Pérot device, where $\omega_{\mathrm{FSR}}=c / 2 d$, with $c$ the speed of light and $d$ the spacing of the mirrors. The resolution is determined from the ratio of the free spectral range, to the full width at half maximum in intensity (FWHM) of the Rayleigh peak. The contrast in one pass is approximately $10^{3}$, while in five-pass it can be as high as $10^{10}$. Both the measured finesse and contrast are lower than the theoretical expectations of 62 and $6 \times 10^{11}$, respectively. This may be due to dust on the mirror surfaces, which produces extra scattering, and hence lowers the contrast.

The most significant source of error in the Brillouin measurements arises from the uncertainty in the determination of the scattering angle $\theta$. An additional possible source of error arises in the determination of the $\omega_{\mathrm{FSR}}$, which involves the precise knowledge of the spacing $d$. This latter error can be significant when $d$ is small. To minimize our experimental error, we calibrate the system by measuring $v$ using some simple liquids for which the sound velocities are well known. These liquids include acetone, methanol, and distilled water. The calibration procedure is used to determine both the plate spacing $d$ and the scattering angle $\theta$. To determine $d$, we first measure the velocity of the calibration fluids with $\theta$ near $180^{\circ}$, where $q$ is insensitive to $\theta$. Once the $\omega_{\mathrm{FSR}}$ is determined, we then align the optics with the desired scattering angle, and measure the sound velocities from the three simple liquids again. This enables the scattering angle to be determined. With both the $\omega_{\text {FSR }}$ and $\theta$ calibrated using this procedure, the remaining uncertainty is from the determination of the peak positions in the spectra. Even with this calibration procedure, the systematic relative error in determining $v$ can be as high as $\simeq 0.07$. However, the relative error for measurements of $v$ as a function of $\phi$ at a fixed angle is substantially less because all the calibration remains unchanged. We estimate that the relative error is about 0.01 , primarily due to the uncertainty in determining the positions of the Brillouin peaks.

The damping of sound $\Delta v$ is measured from the full 
width at half maximum $\Delta \omega_{\text {meas }}$ of the Brillouin peak after accounting for the system resolution $\Delta \omega_{\text {sys. }}$. The simplest way to deconvolute the system resolution is to use the approximate relation $\Delta \omega_{\text {meas }}^{2}=\Delta \omega_{\text {sys }}^{2}+\Delta \omega^{2}$, which assumes that both the Brillouin peak and the system resolution function are Gaussian in shape. When the width is significantly broader than the system resolution, this approximation gives reliable results. However, when the measured width is comparable to the resolution, the results are less reliable.

We have also carried out more precise fitting of the spectra for some experiments, where theory is available. This requires a convolution of the theoretical spectrum with the instrumental response to calculate the measured spectrum

$$
I_{\mathrm{obs}}(\omega)=A \int_{-\infty}^{\infty} I_{\mathrm{ins}}\left(\omega^{\prime}\right) f\left(\omega-\omega^{\prime}\right) d \omega^{\prime} .
$$

Here, $I_{\text {obs }}(\omega)$ is the measured spectrum. $A$ is an overall intensity normalization, $f(\omega)$ is the theoretical form for the spectrum, and

$I_{\mathrm{ins}}(\omega)=\left(\frac{1}{1+\left(\omega_{\mathrm{FSR}} / \pi \Delta \omega_{\mathrm{sys}}\right) \sin \left(\pi \omega / \omega_{\mathrm{FSR}}\right)}\right)^{p}$

is the instrumental response function [2]. The instrumental response is obtained experimentally by using an extended source obtained from scattering from a piece of paper. We fit the measured response to Eq. (4b) to obtain both the width of the Rayleigh peak $\Delta \omega_{\text {sys }}$ and the exponent that is determined by the number of passes $p$. While we should have $p=5$, we typically obtain better fits using $p \simeq 3$. 8. This then provides a functional form to describe the instrumental response. Our measured finesse is typically $\simeq 50$, which is less than the theoretical reflectivity finesse of 62 . The discrepancy is due in part to other effects which degrade the finesse, including the mirror flatness and the pinhole size.

To determine the frequency dispersion of the sound velocity, we use an ultrasonic technique to obtain lowfrequency measurements. In this technique, a transducer is used to excite a pulse of the sound waves in the megahertz range, and the velocity is determined by the measurement of the time $t_{0}$ required for the pulse of sound to travel through a cell of length $L$. These measurements are done either by measuring the time period between two echoes or by using a delay line technique.

In our experiment, the $\mathrm{cw}$ signal obtained from a frequency synthesizer is divided into two parts by a power divider. One part is fed into a gate amplifier where a pulse of length $\sim 0.03 \mu \mathrm{sec}$ is formed. This is used to drive a transducer at its resonant frequency to generate the ultrasonic sound waves in the fluid in the sample cell, which is about $10 \mathrm{~mm}$ in length. A second transducer, mounted on the other side of the cell, serves as the receiver. The time of flight of the sound can be measured directly using the receiver to determine the transit time $t_{0}$ of the pulse. Alternatively, small changes in the velocity can be determined very accurately using a delay line technique. The second part of the $\mathrm{cw}$ signal is fed into another gate amplifier, forming a second pulse. This pulse is delayed by a known amount and is combined with the signal from the receiver. Small changes in $v$ will cause a phase shift in the delayed pulse compared with the transmitted pulse. This phase shift is determined by adjusting the delay line to match the phase of two pulses, so that they null each other in the detector. By measuring the time difference required for matching, the change in the transit time $\Delta t$ can be determined with a relative accuracy as high as $10^{-3}$.

We first use the time-of-flight measurement to determine $t_{0}$ for the solvent. Then the volume fraction of micelle or microemulsion droplets is varied in steps of about 0.025 , and the change in the transit time $\Delta t$ is measured using the delay line. The velocity is then calculated from

$$
v(\phi)=\frac{L}{t_{0}+\Delta t} .
$$

To measure the low-frequency dispersion in the velocity, we use transducers of $2,3,10$, and $15 \mathrm{MHz}$. Data at 6 $\mathrm{MHz}$ are also obtained using the third overtone of the 2$\mathrm{MHz}$ transducer. We also tried to measure the signal at $45 \mathrm{MHz}$ using the third overtone of the $15-\mathrm{MHz}$ transducer, but the damping of the sound is too strong to detect the signal for $\phi>0.3$ with our cell.

The length of the sample cell $L$ is calibrated by measuring $v$ for simple liquids, including acetone, methanol, and distilled water, where the velocity of sound is well known. The main experimental error in the absolute value of $v$, therefore, arises from the measurement of $t_{0}$, and is typically $\delta t_{0} / t_{0} \simeq 0.01$. However, the relative error in measuring the $\phi$ dependence of $v$ at each frequency is at least an order of magnitude lower, as the phase matching technique is used.

The damping of sound is measured by the decay in the amplitude of the first or second echo of the pulse in the cell, $A(\phi)=A_{0} e^{-\alpha(\phi) L}$, where $\alpha$ is the attenuation coefficient of sound, and $A_{0}$ is the amplitude of the initial pulse. We also calibrate $A_{0}$ using simple liquids. However, due to the uncertainty in the amplitude measurements, the reliability in the value of $\alpha$ is reduced.

\section{RESULTS AND DISCUSSION}

The experimental results will be presented and discussed in this section. We summarize our main results in Table I, where the systems and their elastic properties are listed. We first discuss the results of the velocity measurements for micelles. By varying the carbon number of solvent oils, the frequency dispersion of the sound velocity is changed due to the change in the magnitude of the attractive interaction. Next, we discuss the results for microemulsions, where we vary the droplet sizes as well as the solvents. The competition between the effects of the interaction energy and the intrinsic stiffness of the droplet phase determines the observed behavior in these systems. We then discuss the dynamic rigidity percolation, which accounts for the pronounced viscoelastic behavior observed for both the micelle and microemulsion systems. Results for the calculation of the Brillouin spectra are then discussed in conjunction with the theoretical model used to fit the spectra. Finally, we end this section by discussing an additional high-frequency dispersion of 
TABLE I. Summary of properties of AOT micelle and microemulsion systems.

\begin{tabular}{|c|c|c|c|c|c|c|c|}
\hline \multirow[b]{2}{*}{ Properties } & \multicolumn{3}{|c|}{ Inverted Micelles } & \multicolumn{4}{|c|}{ Microemulsions } \\
\hline & Hexane & Decane & Hexadecane & Hexane & & Decane & \\
\hline Size $(r)$ & & $15 \AA$ & & $25 \AA$ & $25 \AA$ & $45 \AA$ & $75 \AA$ \\
\hline$\phi$ dispersion & yes & yes & yes & yes & yes & yes & yes \\
\hline$f$ dispersion & no & yes & yes & no & no & no & no \\
\hline$f_{c}$ & & $\sim 10^{8}$ & $<10^{6}$ & $\sim 10^{8}$ & $<10^{6}$ & $<10^{6}$ & \\
\hline$v(\mathrm{~m} / \mathrm{s})(\phi=0.5)$ & 1180 & 1245 & & 1116 & & & \\
\hline$v_{\infty}(\mathrm{m} / \mathrm{s})(\phi=0.5)$ & & 1300 & & 1138 & 1285 & 1280 & \\
\hline$\mu_{d}\left(\mathrm{~N} / \mathrm{m}^{2}\right)$ & & $\sim 10^{8}$ & $\sim 2 \times 10^{8}$ & $1.8 \times 10^{8}$ & $1.5 \times 10^{8}$ & $1.0 \times 10^{8}$ & \\
\hline
\end{tabular}

the sound velocity observed for both the micelle and microemulsion systems, which we interpret as a relaxation of the tails of the surfactant molecules themselves.

\section{Micelles}

In inverted micelles, the solid core formed by the close-packed head groups of the AOT molecules is more rigid than the suspending fluid. Thus, with increasing volume fraction of micelles, the sound velocity increases [6]. We illustrate this behavior in Fig. 2, which shows the $\phi$ dependence of $v$ measured at different frequencies for micelles in different solvents: hexane $\left(\mathrm{C}_{6} \mathrm{H}_{14}\right)$, decane $\left(\mathrm{C}_{10} \mathrm{H}_{22}\right)$, and hexadecane $\left(\mathrm{C}_{16} \mathrm{H}_{34}\right)$. The results shown were obtained using both ultrasonic measurements and Brillouin scattering. These measurements allow us to probe the effects of the different interaction energies between the droplets on the frequency dependence of the elastic properties.

There is a great deal of information contained in Fig. 2. The sound velocity exhibits a strong dependence on the

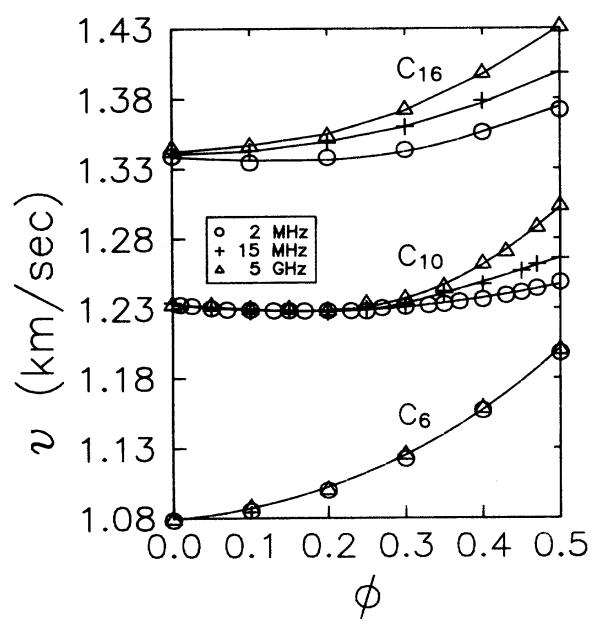

FIG. 2. Velocity of sound in AOT inverted micelles as a function of volume fraction for several different solvents: hexane $\left(C_{6}\right)$, decane $\left(C_{10}\right)$, and hexadecane $\left(C_{16}\right)$. Ultrasonic measurements were used to obtain the 2- and $15-\mathrm{MHz}$ data; Brillouin scattering was used to obtain the high-frequency data. The solid lines are the fits with the effective-medium theory. solvent molecule, increasing as the alkane number increases. The sound velocity also increases substantially as a function of $\phi$ for all cases. The amount of dispersion in frequency also changes substantially as the chain length of the solvent is increased. For hexane, there is no frequency dispersion of the sound velocity within the frequency range measured for all $\phi$. For decane, there is a pronounced dispersion with frequency at higher volume fractions. For hexadecane, there is frequency dispersion at all $\phi$.

The solid line through the hexane data is the fit using Eq. (3), the simple model which does not include any shear modulus. Excellent agreement is obtained. From the fit, we obtain the elastic constants of the droplet phase, $\beta_{d}^{\prime} \simeq 1.33 \times 10^{9} \mathrm{~N} / \mathrm{m}^{2}$ and $\beta_{d}^{\prime \prime} \simeq 33.58 \times 10^{6} \mathrm{~N} / \mathrm{m}^{2}$. The absence of a shear modulus implies that the relaxation time is very fast due to the smaller interaction energy between droplets. Therefore, the critical frequency $f_{c}$ for the system is too high to be seen in our experimentally accessible range, and must be greater than $10 \mathrm{GHz}$.

As we increase the alkane number of the solvent by using decane, the data exhibit a pronounced frequency dispersion for $\phi \geq 0.16$. We are able to use the isolated droplet model to describe the $\phi$ dependence of the velocity for the low-frequency measurements, as shown by the solid line through the $2-\mathrm{MHz}$ data. The excellent fit indicates that the system does not support shear at low frequencies. However, if we apply the same model to the higher-frequency data, we are unable to fit the data, since the velocity increases so much more rapidly at higher $\phi$. Therefore, we must use Eq. (2), which includes a contribution from the shear modulus of the surfactant phase to the elastic properties of the system. The solid lines through the $15-\mathrm{MHz}$ and $4-\mathrm{GHz}$ decane data in Fig. 2 are the results of the fit using the symmetric model. The fit gives $\mu^{\prime} \sim 10^{8} \mathrm{~N} / \mathrm{m}^{2}$ for the composite system at 5 $\mathrm{GHz}$. The fitting parameters of the surfactant micelle phase are $\beta_{d}^{\prime} \simeq 1.62 \times 10^{9} \mathrm{~N} / \mathrm{m}^{2}$ at $2 \mathrm{MHz}$, increasing to $\simeq 1.98 \times 10^{9} \mathrm{~N} / \mathrm{m}^{2}$ at $5 \mathrm{GHz}$, and $\beta_{d}^{\prime \prime} \simeq 6.0 \times 10^{6} \mathrm{~N} / \mathrm{m}^{2}$ at $2 \mathrm{MHz}$, increasing to $\simeq 7.0 \times 10^{7} \mathrm{~N} / \mathrm{m}^{2}$ at $5 \mathrm{GHz}$. We note that the fits are very sensitive to the value of $\beta_{d}^{\prime}$, enabling a precise determination of its value. By contrast, $\beta_{d}^{\prime \prime}$ cannot be determined as accurately because of the greater error involved in our measurements of the damping of the sound velocity. The frequency dependence of the real part of the shear modulus of the surfactant phase $\mu_{d}^{\prime}$ has a Debye relaxation form, with a characteristic frequency 
of $f_{c} \simeq 10^{8} \mathrm{~Hz}$ [6]. The imaginary part of the shear modulus $\mu_{d}$ varies from $10^{5}$ to $10^{7} \mathrm{~N} / \mathrm{m}^{2}$ within the measured frequency range. The increase of $\beta_{d}$ with frequency indicates that the stiffness of the droplet phase increases due to the formation of random networks, which contribute an additional shear modulus $\mu_{d}$.

When we further increase the alkane number of the solvent by using hexadecane, an even larger velocity dispersion in frequency is seen, as shown in Fig. 2. In this case, however, the dispersion exists for all volume fractions. Indeed, even the hexadecane itself $(\phi=0)$ exhibits a slight frequency dispersion. To fit these data, we must use Eq. (2) at all frequencies, implying that for hexadecane, $f_{c}$ has decreased to below $1 \mathrm{MHz}$. We find that $\mu_{d}$ increases slightly from $\simeq 2 \times 10^{8} \mathrm{~N} / \mathrm{m}^{2}$ at $2 \mathrm{MHz}$ to $2.2 \times 10^{8} \mathrm{~N} / \mathrm{m}^{2}$ at $5 \mathrm{GHz}$, which means that the droplets form networks which support shear even at the lowest frequencies that we measure. The fitting parameters $\beta_{d}^{\prime}$ increases from $1.97 \times 10^{9}$ to $2.02 \times 10^{9} \mathrm{~N} / \mathrm{m}^{2}$ as the frequency increases from $2 \mathrm{MHz}$ to $5 \mathrm{GHz}$, and the imaginary part of the shear modulus $\mu_{d}^{\prime \prime}$ increases from $1 \times 10^{6}$ $\mathrm{N} / \mathrm{m}^{2}$ at $2 \mathrm{MHz}$ to $3 \times 10^{6} \mathrm{~N} / \mathrm{m}^{2}$ at $5 \mathrm{GHz}$.

The observed variation of the characteristic frequency shows that the viscoelastic behavior of the micelles is directly related to the characteristics of the oil used as the solvent. The decrease in $f_{c}$ with the carbon chain length of the solvents results from the increase in the magnitude of the attractive interaction between the droplets [10]. As the carbon chain length increases, it becomes increasingly favorable for the surfactant tails of two neighboring droplets to interpenetrate as compared to solvent molecules, filling the spacing between the tail groups. As a consequence, the energy of the attractive interaction increases, with a concomitant decrease in $f_{c}$. The change in $f_{c}$ does not simply reflect the change in the viscosity of the oil, which might be expected if $f_{c}$ were determined by Brownian motion of the micelles, since the diffusion coefficient is inversely proportional to the viscosity. The viscosities for hexane, decane, and hexadecane are $0.7,1.3$, and $3.1 \mathrm{cp}$, respectively [13], so the diffusion coefficients of the micelles change by significantly less than a decade on going from hexane to decane to hexadecane. By contrast, $f_{c}$ changes by several orders of magnitude on going from hexane to decane and to hexadecane. Thus the interaction time, and hence $f_{c}$, must be determined by the attractive interaction energies rather than the Brownian diffusion times of the micelles.

\section{Microemulsions}

Another way to vary the interaction energy, and hence $f_{c}$, is to change the size of droplets by swelling them with water to form microemulsions. This increases the radius of curvature, and thereby increases the area of overlapping tails for two interacting droplets, leading to an increase in the interaction energy. To investigate these effects, we show, in Fig. 3, the results of $v$ as a function of $\phi$ for microemulsions in decane, using droplets in three different radii, 25,45 , and $75 \AA$. The data were obtained

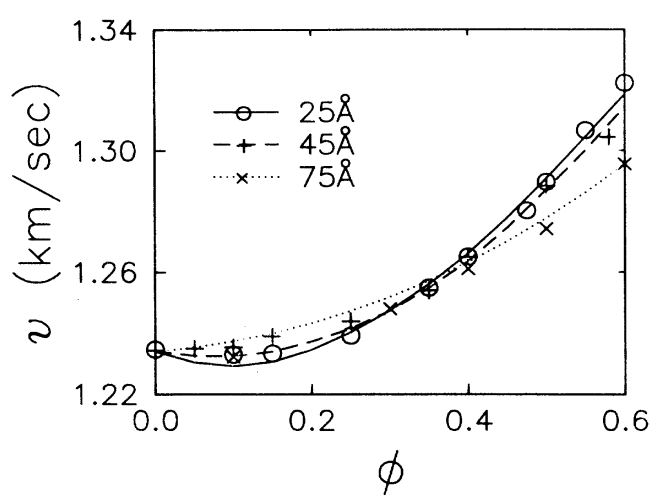

FIG. 3. The velocity of sound for AOT-decane-water microemulsions of three sizes as a function of volume fraction. The solid lines represent fits to the effective-medium theory.

at a frequency of $5 \mathrm{GHz}$ using Brillouin scattering. The velocities for each of the three systems increase with increasing $\phi$. The lines through the data represent the results of fits using the effective-medium theory. For the two microemulsions with smaller sizes, the data must be fit using Eq. (2), which includes a contribution from the shear modulus due to the networks formed by the microemulsion phase. The values of $\mu_{d}^{\prime}$ from fits are $\sim 1.5 \times 10^{8}$ and $1.0 \times 10^{8} \mathrm{~N} / \mathrm{m}^{2}$ for the 25 -and $45-\AA$ radius samples, respectively. By contrast, for the microemulsions with $75-\AA$ radius, the data are fit using the simpler model, without shear. These results suggest that the interaction between microemulsions due to the overlapping of the tails again leads to the formation of networks at $5 \mathrm{GHz}$. For microemulsions with small size, the shear modulus due to the network results in an increase of the longitudinal elastic modulus at high $\phi$. However, as the microemulsion droplets grow in diameter, the increasing volume of water compared to surfactant reduces the intrinsic rigidity of droplets themselves. Thus the shear modulus of the droplet phase decreases again as the droplet size grows.

We also investigated the frequency dispersion of the sound velocity in the microemulsions by measuring the dependence of $v$ on $\phi$ using ultrasonics from 2 to 30 MHz. Virtually no additional frequency dispersion was observed for all volume fractions for any of these microemulsion samples. These observations suggest that the increased contact area, resulting from the larger radius of curvature of microemulsions, causes the interaction energy to increase. Thus $f_{c}$ is decreased, so that $f \gg f_{c}$ for all the frequencies used here.

To confirm this picture, we can exploit the behavior observed for the micelles, and change the interaction energy by varying the chain length of the hydrocarbon solvent. In order to bring the characteristic frequency into an experimentally accessible region, we must decrease the interaction energy between droplets, which requires the use of a solvent with a shorter chain length. Thus we repeat the measurements for the 25 - $\AA$-radius microemulsions using hexane as the solvent. As expected, consider- 


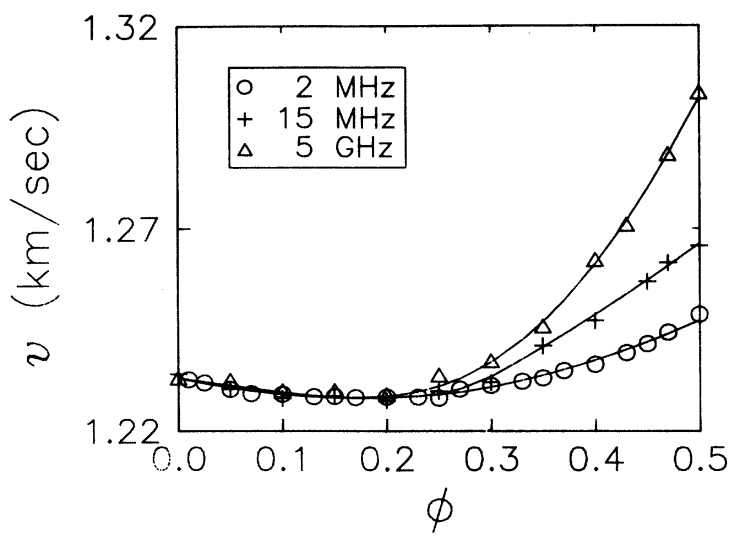

FIG. 4. Velocity of sound in AOT-hexane-water microemulsions as a function of volume fraction for different frequencies. The droplet sizes are $25 \AA$ in radius. The solid lines represent fits to effective-medium theory.

able additional frequency dispersion is indeed observed. This is illustrated in Fig. 4, where we show the sound velocity as a function of droplet volume fraction measured at three different frequencies. These results exhibit a striking similarity to those obtained for the micelles in decane. The effective-medium theory fit to the $2-\mathrm{MHz}$ data requires a shear modulus near zero for the microemulsion phase. By contract, the fits to the data at the highest frequency yield a much larger shear modulus, $\mu_{d} \simeq 1.8 \times 10^{8} \mathrm{~N} / \mathrm{m}^{2}$. In Fig. 5 we plot the velocity as a function of frequency for several different volume fractions. The behavior is again similar to that observed for the micelles in decane [6]; an additional increase in $v$ as the frequency increases, with the amount of the increase becoming larger at higher $\phi$. In all cases, however, the asymptotic values of $v$ are approached at both high and

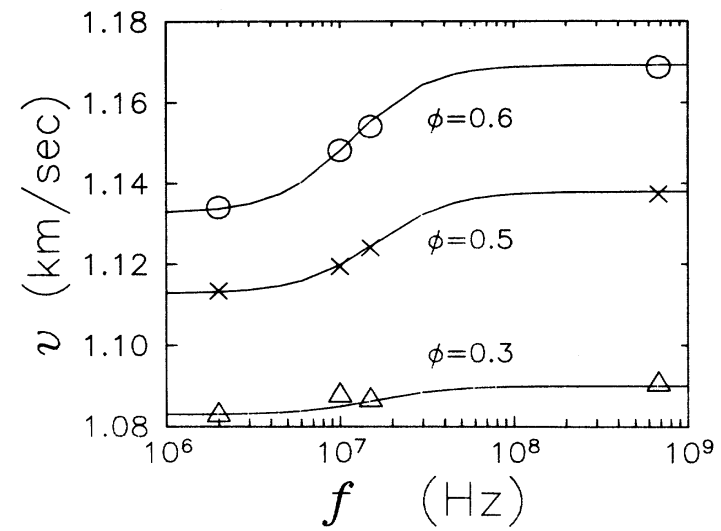

FIG. 5. Frequency dispersion of the sound velocity of $25-\AA$ microemulsions in hexane at three volume fractions: $\phi=0.3$, 0.5 , and 0.6. The solid lines are single Debye relaxation with $\tau \simeq 1 \times 10^{-8} \mathrm{sec}$. The additional increase of $v$ at high frequency is a function of $\phi$, which implies an increase in the elastic modulus due to the droplet networks. low frequencies. The solid line represents fits to a Debye model with a single relaxation frequency, $f_{c} \sim 10^{8} \mathrm{~Hz}$.

The observed viscoelastic behavior in microemulsion systems is consistent with our physical picture: the attractive interaction between the droplets leads to the formation of extended networks. Provided the water cores of the droplets are sufficiently small to ensure the necessary rigidity for the droplet phase, the networks can support shear at high frequencies, $f>f_{c}$. Therefore, we see a transition in the behavior of the system from a viscous liquid at low frequencies to an elastic solid at high frequency due to the random networks formed by the droplet phase.

\section{Dynamic rigidity percolation}

The dynamics of the inverted micelles or microemulsions lead to their unique behavior. At low frequencies their diffusive motion ensures that the droplets behave as independent, isolated spheres even at high $\phi$. By contrast, at higher frequencies, the attractive interaction between the droplets ensures that random, extended networks are formed for times long compared to the period of the sound. The shear moduli of networks thus supply the added rigidity to the system, which leads to an increase in sound velocity. The fact that they behave as fluids at low frequencies ensures a truly random dispersion of the droplets. The fact that we can measure the speed of sound at both low and high frequencies means that we can distinguish the additional contribution of the rigidity of the extended networks. Thus, these are ideal systems to use to measure the elastic properties of random inhomogeneous mixtures. Here we will show that, for both the inverted micelles in decane and the microemulsions in hexane, the contribution of the shear modulus to the elastic modulus of the system displays a scaling behavior indicative of dynamic rigidity percolation [7].

The difference between the high- and low-frequency moduli $\Delta \beta^{\prime}$ is, to lowest order, linear in the change in $\mu^{\prime}$, and thus provides a direct measure of the rigidity of the micelle network at each $\phi$. We can determine $\Delta \beta^{\prime}$ directly from the velocity measurements by measuring the asymptotic values of $\beta^{\prime}$ at high and at low frequencies, and taking the difference. For the inverted micelles in decane, we are able to discern a change in $v$ only at volume fractions above $\phi \simeq 0$.16. Thus we plot $\Delta \beta^{\prime}$ as a function of $\phi-\phi_{c}$, with $\phi_{c} \simeq 0.16$, as the open circles in the logarithmic plot in Fig. 6. The resolution of the Brillouin peaks precludes measurements closer to $\phi_{c}$. We observe a clear power-law behavior, with a rigidity exponent of $\tau \simeq 2$.5. This scaling extends over a rather large range in $\phi-\phi_{c}$, and is consistent with a random percolating network. We emphasize that this observation is based solely on the experimental data, independent of any model. However, this is a dynamic rather than a static measurement. This limits the spatial extent of the sensitivity of this measurement of the rigidity to roughly the wavelength of the sound, which is nonetheless at least 100 times larger than the single droplet diameter, even at the highest frequency. Finally, this rigidity transition occurs 


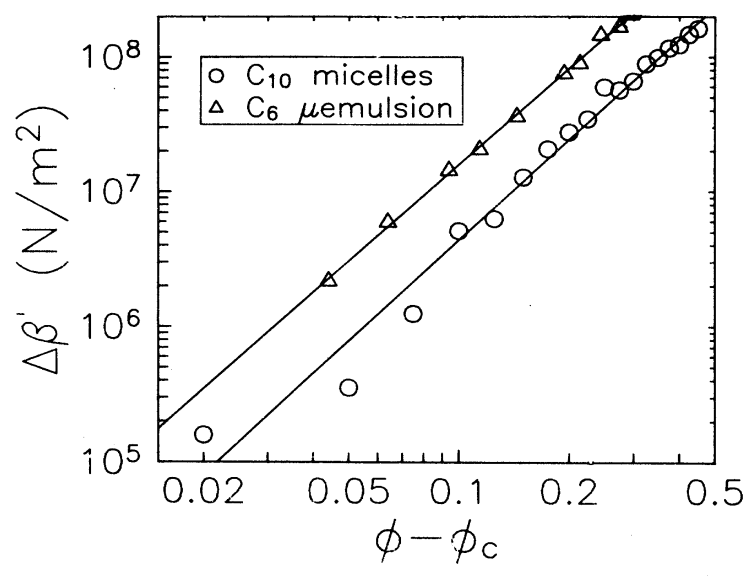

FIG. 6. The additional elastic modulus $\Delta \beta^{\prime}$ at high frequencies as a function of the volume fraction of droplets for both micelles in decane and microemulsions in hexane, showing the critical behavior of the contribution of the shear modulus at high frequencies. The solid lines are calculations of $\left(\phi-\phi_{c}\right)^{t}$ with $t \simeq 2.5$, and $\phi_{c}=0.16$ for micelles and 0.15 for microemulsions.

only for the surfactant micelle phase, which forms the random connected network. The surrounding oil phase ensures that the mixture maintains a finite bulk modulus at all $\phi$ and does not exhibit a percolation threshold.

Since the behavior of $25-\AA$-radius microemulsion in hexane is very similar to that seen for micelles in decane, we can also look for a scaling behavior indicative of dynamic rigidity percolation. In Fig. 6, we also plot the $\Delta \beta^{\prime}$, for the $25-\AA$ microemulsion in hexane as a function of $\phi-\phi_{c}$. Scaling behavior is again observed. The solid line is a fit to $\Delta \beta^{\prime} \sim\left(\phi-\phi_{c}\right)^{\tau}$, where we obtain $\phi_{c} \simeq 0.15$ and $\tau \approx 2$.5. Both parameters are in excellent accord with those of the micelle data [6]. Thus we observe a dynamic rigidity percolation for the microemulsion system as well, provided we adjust the solvent to bring $\tau_{c}$ into a measurable range.

There are relatively few results available for elasticity percolation of random networks in three dimensions with which these data can be compared. No calculations for the elastic properties of random, multiply connected three-dimensional networks exist. The electrical conductivity [20] of three-dimensional random networks is believed to scale as $\sigma=\left(\phi-\phi_{c}\right)^{t}$, with $t=2$. It has been conjectured that the elasticity exponent has the same scaling as the conductivity exponent [21]. This is true, however, only if central forces alone exist between the constituent particle in the network [22]. By contrast, noncentral forces, or bond-bending forces, break the similarity between conductivity and elasticity, leading to an increase in the elasticity exponent [23]. For singly connected networks [24], the exponent is predicted to be about 3.5. Experimentally, measurements on beams of sintered metal [25] yielded $\tau \simeq 3.8$, and $t \simeq 2.0$, over an equally large range in $\phi-\phi_{c}$. The behavior of the shear rigidity of the network of surfactant micelles is consider- ably different. This may reflect the different nature of the micelle interactions, which might change $\tau$. Finally, the value of $\phi_{c}$ found here is consistent with that found from simulations of interacting spheres [26]. It can be expected to change as $E_{a}$ is varied, modifying $\tau_{c}$. These inverted micelles allow the investigation of this important issue.

One final interesting comparison that can be made is between the dynamic rigidity percolation behavior reported here and the conductivity percolation behavior that is also exhibited by the microemulsions [27]. The critical volume fraction for conductivity percolation is found experimentally to be strongly dependent on the droplet size [16], the chain length of the solvent oil [10], and the temperature as a critical point is approached [10]. While the precise origin of this behavior is still subject to some speculation, it is generally believed that the mechanism for conductivity in microemulsions involves the hopping of charge between the droplets [28]. The hopping range varies with droplet size, solvent oil, and temperature, accounting for the observed variation in $\phi_{c}$. By contrast, dynamic rigidity percolation depends on the formation of a connected network that is mechanically rigid and can support shear. Furthermore, this rigidity percolation is dynamic, and here we investigate only the limit at very high frequencies, where the rigid, percolating network remains fully formed during the measurement. As such, dynamic rigidity percolation is somewhat different from conductivity percolation. It would, however, be of interest to further investigate the behavior of $\phi_{c}$ for dynamic rigidity percolation to explore its sensitivity to both frequency and the strength of the interaction potential. In the experiments reported here, we have adjusted the interaction energy of both systems to ensure that $f_{c}$ lies in an experimentally accessible range. As such, we find that $\phi_{c}$ is the same for each system. In addition, we were unable to detect any dependence of either $\phi_{c}$ or $\tau$ on frequency as the characteristic frequency was approached. Further experiments to determine the sensitivity of $\phi_{c}$ on interaction energy and on frequency would be of great interest, and would provide a more complete comparison of the behavior of conductivity percolation with that dynamic rigidity percolation.

\section{Spectral fits}

There is considerably more information in the full Raleigh-Brillouin spectra than the peaks and widths of the Brillouin doublet. For simple liquids, the spectrum consists of three Lorentzian lines. However, for molecular fluids, an additional, prominent continuous background is found between the Rayleigh and the Brillouin doublet $[1,29,30]$. This additional scattering results from the internal degree of freedom of the molecules, which couples to the compressional motion, and thus contributes scattered light intensity [1]. The Brillouin spectra of the micelles and microemulsions also show significant intensity in the region between the Rayleigh and Brillouin peaks. We might expect the relaxation of the extended networks to produce this broad background in a manner analogous to the internal relaxation of simpler molecules. 
To test this hypothesis, we employ Mountain's formalism [1] to compare to our measured spectra.

To account for the contribution of the molecular relaxation, the viscosity must be made to be frequency dependent $[1,12]$.

$$
\eta_{V}(\omega)=\eta_{V}+\frac{\left[v_{\infty}^{2}-v^{2}(\omega)\right] \tau_{c}}{1+i \omega \tau_{c}}
$$

where $\eta_{V}$ is the center-of-mass part of the bulk viscosity at constant volume $V$ and is frequency independent, $\tau_{c}$ is the relaxation time, and $v_{\infty}$ is the sound velocity in the high-frequency limit. The frequency dependence of $\eta_{V}(\omega)$ is such that for $\omega$ small compared to the relaxation rate $1 / \tau_{c}, \eta(\omega) \rightarrow \eta_{V}+\left[v_{\infty}^{2}-v^{2}(\omega)\right] \tau_{c}$; for frequencies large compared with $1 / \tau_{c}, \eta_{V}(\omega) \rightarrow \eta_{V}$. Thus, $\eta_{V}(\omega)$ provides a phenomenological description of the effects of the molecular relaxation process on the viscosity. The Rayleigh-Brillouin spectrum for constant scattering wave vector $q$ is given by $[1,30]$

$$
I(\omega)=\operatorname{const} \frac{N_{1}(\omega) D_{1}(\omega)+N_{2}(\omega) D_{2}(\omega)}{D_{1}^{2}(\omega)+D_{2}^{2}(\omega)},
$$

where

$$
\begin{aligned}
N_{1}(\omega)= & -\omega^{2}+\gamma D_{T} D_{V} q^{4}+v^{2} q^{2}(1-1 / \gamma) \\
& +\left(\gamma D_{T} b_{1}^{\prime} q^{4}+b_{1}^{\prime} \omega^{2} q^{2} \tau_{c}\right) /\left(1+\omega^{2} \tau_{c}^{2}\right), \\
N_{2}(\omega)= & \omega\left[\gamma D_{T} q^{2}+D_{V} q^{2}\right. \\
& \left.+\left(b_{1}^{\prime} q^{2}+\gamma D_{T} b_{1}^{\prime} \tau_{c} q^{4}\right) /\left(1+\omega^{2} \tau_{c}^{2}\right)\right], \\
D_{1}(\omega)= & -\omega^{2}\left(\gamma D_{T} q^{2}+D_{V} q^{2}\right)+v^{2} q^{4} D_{T} \\
& +\left(\gamma D_{T} b_{1}^{\prime} \omega^{2} q^{4} \tau_{c}-b_{1}^{\prime} \omega^{2} q^{2}\right) /\left(1+\omega^{2} \tau_{c}^{2}\right), \\
D_{2}(\omega)=\omega & {\left[-\omega^{2}+v_{2} q^{2}+\gamma D_{T} D_{v} q^{4}\right.} \\
& \left.+\left(b_{1}^{\prime} q^{2} \omega^{2} \tau_{c}+\gamma D_{T} b_{1}^{\prime} q^{4}\right) /\left(1+\omega^{2} \tau_{c}^{2}\right)\right],
\end{aligned}
$$

and where

$$
b_{1}^{\prime}=\frac{\left(v_{\infty}^{2}-v^{2}\right) \tau_{c}}{\rho}
$$

In these equation, $D_{T}=\lambda / \rho C_{p}$ is the thermal diffusivity and $\lambda$ is the thermal conductivity, $D_{V}=\left(\eta_{V}+\frac{4}{3} \eta_{S}\right) / \rho$ is the longitudinal kinematic viscosity, with $\eta_{V}$ and $\eta_{S}$ the bulk and shear viscosities, respectively, and $\gamma=C_{p} / C_{V}$, where $C_{p}$ and $C_{V}$ are the specific heats at constant pressure and volume, respectively.

The exact expression for the Brillouin spectrum with the structural relaxation, Eq. (7), is simplified for small viscosity, such the $D_{V} q^{2}<<\omega(q)$, where $\omega(q)$ is the frequency of the Brillouin shift. The spectrum is comprised of three Lorentzian peaks: the first is the Rayleigh peak, the second is the pair of Brillouin peaks, while the third is a broad peak centered at zero frequency [28]. The width of the Rayleigh peak depends on the thermal diffusivity. The Brillouin doublet has a frequency shift of $\omega= \pm v q$, while its width is determined primarily by $D_{V} q^{2}$. The width of the broad peak depends on the structural relaxation time $\tau_{c}^{-1}$, while its magnitude depends on the ratio of $v / v_{\infty}$, as well as on $\tau_{c}$.

Thus to compare the theoretical spectra to our data, there are six unknown parameters, $D_{T}, \eta_{l}, \gamma, \tau_{c}, v$ and $v_{\infty}$. However, because each parameter influences the spectrum in such a different fashion, reliable values for these parameters can be obtained from fits to the data. The only exceptions to this are the values obtained for the Brillouin velocity $v$ and the high-frequency limit of the velocity $v_{\infty}$. Attempts to determine both of these parameters independently from the fits invariably led to unrealistic values, presumably because our data are obtained at rather large frequencies, where $v$ is nearly equal $v_{\infty}$. Thus in fitting the data, we fix $v$, determining its value from the maximum intensity of the Brillouin doublets, and use $v_{\infty}$ as a fitting parameter.

To fit the experimental data, we convolve the theoretical expression, Eq. (7), with the instrumental response function, Eq. (4a), and use a nonlinear least-squares routine to obtain values for $D_{T}, \eta_{l}, \gamma, \tau_{c}$, and $v_{\infty}$, as well as an overall normalization constant to account for the absolute intensity. This theoretical formalism provides excellent agreement with the data, as shown by the examples in Fig. 7, which shows the data for micelles in hexane, decane, and hexadecane, all with $\phi=0.5$ and obtained at a scattering angle of $90^{\circ}$ with $\lambda=5145 \AA$. The intensities are plotted on a logarithmic scale to show the large variation, and the dark counts and background have been subtracted. The Raleigh peaks are all substan-

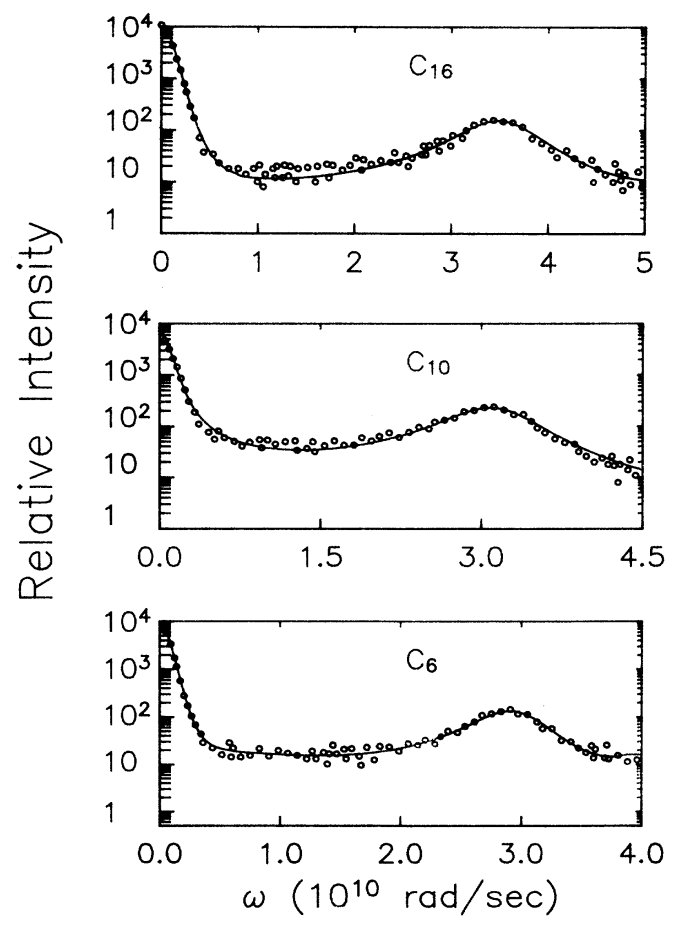

FIG. 7. Brillouin spectra of AOT micelles for several different solvents, hexane $\left(\mathrm{C}_{6}\right)$, decane $\left(\mathrm{C}_{10}\right)$, and hexadecane $\left(C_{16}\right)$ at $\phi=0.5$. The solid lines are the theoretical spectra calculated from Eq. (7). The scattering angle is $90^{\circ}$, and the wavelength is $5145 \AA$. 
tially larger than the Brillouin peaks, as is typically observed for viscoelastic materials. In addition, there is a continuous intensity between the Rayleigh and the Brillouin peaks, reflecting the additional relaxation mechanism.

In all the spectra, the width of the Rayleigh peak is determined by $D_{T} q^{2}$. Since the measured width is close to the limit of the resolution of the interferometer, we are unable to precisely determine the value of $D_{T}$. It is on the order of $10^{-4} \mathrm{~cm}^{2} / \mathrm{sec}$, which is the same order of magnitude as $D_{T}$ determined for decane or $\mathrm{CCl}_{4}$.

The width of the Brillouin peak is determined by $D_{V} q^{2}$. In general, for a viscoelastic fluid, the longitudinal kinematic viscosity $D_{V}$ is frequency dependent, reflecting the consequences of the relaxation processes in the fluid. From the width of the Brillouin peaks for the micelles in decane, we obtain values for $D_{V}$ of $2.3 \times 10^{-2} \mathrm{~cm}^{2} / \mathrm{sec}$ for $\phi=0$ (pure decane) and $0.11 \mathrm{~cm}^{2} / \mathrm{sec}$ for $\phi=0.5$. We have measured the shear viscosity using a viscometer. We find an exponential increase with volume fraction, from $\eta_{s} / \rho=1.1 \times 10^{-2} \mathrm{~cm}^{2} / \mathrm{sec}$ for pure decane to 0.2 $\mathrm{cm}^{2} / \mathrm{sec}$ for $\phi=0.5$. The static viscosity measurements give a value for $\eta_{s}$ that is larger than that obtained from the Brillouin measurements, which include the contribution of both the shear and bulk viscosities. This behavior is often observed in viscoelastic materials and reflects the complexity of the physical processes which contribute to the width of the Brillouin peaks [31]. Physically, we might also expect the static measurements of the shear viscosity to yield a larger value for $\eta_{s}$, since they reflect a macroscopic flow, wherein the micelles must move distances larger than their own size, making the effects of geometric constraints contribute to the viscosity.

The prominent background induced by the relaxation mechanism in the spectrum depends on the relaxation time $\tau_{c}$ and the velocity ratio $v / v_{\infty}$. For micelles in decane and microemulsions in hexane, where continuous backgrounds are observed, the values of $\tau_{c}$ obtained from fits are $\tau_{c} \simeq 1.4 \times 10^{-8} \mathrm{sec}$ for micelles in decane and $\tau_{c} \simeq 2.4 \times 10^{-8} \mathrm{sec}$ microemulsions in hexane. These are in excellent agreement with the values of $\tau$ obtained from the measured frequency dispersion of the velocity fit to a single relaxation time Debye behavior. In addition, the values of $v_{\infty}$ obtained from the spectral fitting of the two systems are also consistent with those obtained from measured frequency dispersions: the fits to the spectra give $v_{\infty} \simeq 1309 \mathrm{~m} / \mathrm{sec}$ for micelles in decane at $\phi=0.5$, and $v_{\infty} \simeq 1140 \mathrm{~m} / \mathrm{sec}$ for the 25 -A-radius microemulsions in hexane with $\phi=0.5$. The values are in excellent agreement with the values of $1298 \mathrm{~m} / \mathrm{sec}$ for the micelles and of $1130 \mathrm{~m} / \mathrm{sec}$ for the microemulsions. When $\omega \tau_{c}>1$, the broad peak becomes very narrow and cannot be distinguished from the Rayleigh peak. Thus for micelles in hexadecane, where $\tau_{c}>10^{-7} \mathrm{sec}$, the broad peak is great1y reduced. Similarly, the broad peak is essentially absent in the hexane spectra in Fig. 7, and the actual value obtained for $\tau_{c}$ from the fit is unreliable. The value obtained is very low, consistent with the absence of any additional dispersion of $v$ with $f$. Finally, for the 75- $\AA$ radius microemulsions in decane, when the swollen water cores reduce the rigidity of the droplet phase, there is again no contribution due to the relaxation of the microemulsion structures, and the spectra are again similar to those of simple fluids where we do not see a broad peak. Thus the presence of the broad peak in the spectra is completely consistent with our frequency dispersion data. As expected from the theory, it is only observed when $\tau_{c} \sim 10^{-8} \mathrm{sec}$, and when it is present, the value for $\tau_{c}$ obtained from the fit to the spectra is consistent with that obtained directly from the frequency dispersion of $v$.

The Landau-Placzek ratio $\gamma-1$ is the intensity ratio of the Rayleigh peak to the Brillouin doublet. As a fitting parameter, we find $\gamma \simeq 1.2$ for decane, consistent with $\gamma=1.219$ measured for $n$-hexadecane by Patterson and Lindsey [32]. We find that $\gamma$ increases up to 1.8 at high $\phi$ in the micelle systems. However, the values of $\gamma$ obtained from the fits to the spectra of the microemulsion systems, at larger droplet sizes and higher $\phi$, can be as large as 10. This reflects the viscoelastic nature of the system. Similar behavior is found in polymer fluids [33], where the intensity ratio of the central peak to the Brillouin doublet is often much greater than unity.

We also attempted to use a model for a binary mixture [1] to fit our spectra. The binary-mixture model accounts for additional concentration fluctuations which contribute to the density fluctuations. Thus the central component consists of a superposition of two peaks, one resulting from entropic fluctuations, and the other from concentration fluctuations. We find that this model cannot adequately describe our results. The calculated Rayleigh peak from the model is too broad to fit our spectrum, implying that concentration fluctuations of the micelles or microemulsions do not play an important role in the scattering from these systems.

In summary, the spectral fitting using Mountain's relaxation model successfully describes our data. The coupling between the translational motion and the structural rearrangement motions leads to a new nonpropagating mode, with a width on the order of $1 / \tau_{c}[1,31]$. For the micelle and microemulsion systems, the parameter $\tau_{c}$ reflects the structural relaxation time in the system. In addition, the relaxation time and high-frequency sound velocity obtained from the spectral fitting are consistent with those given by the Debye relaxation model fit to the measured frequency dispersion of the sound velocity. Finally, the computed curves are in good agreement with the measured spectra. Thus, a totally self-consistent picture emerges.

\section{Relaxation of surfactant molecule tail groups}

Here we discuss an additional frequency dispersion of the sound velocity found in all the micelle and microemulsion systems. Very near the backward-scattering configurations, where the sound velocities are measured at the highest frequencies, we find that the sound velocity increases rapidly within a very-short-frequency range, regardless of solvent and droplet sizes. We interpret this behavior as a further relaxation process of the surfactant tails themselves.

The experimental results are shown in Fig. 8, where we 


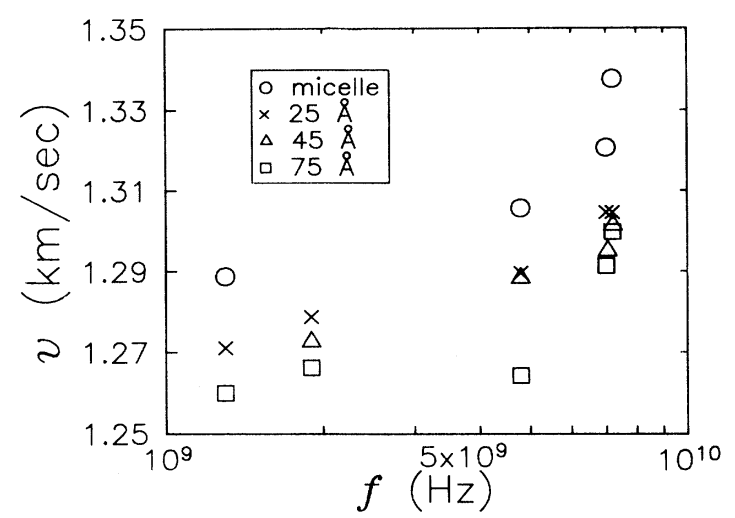

FIG. 8. Additional frequency dispersion of the sound velocity for AOT micelles and microemulsions of three sizes, all in decane and all at $\phi=0.5$, demonstrating the additional relaxation of the tails of the AOT molecules themselves.

plot the dependence of $v$ on frequency for micelles and for three sizes of microemulsions, with radii 25,45 , and $75 \AA$, all at $\phi=0.5$ and all in decane. The data are obtained by varying the scattering angle from $30^{\circ}$ to nearly $180^{\circ}$, using an $\mathrm{Ar}^{+}$laser at $5145 \mathrm{~A}$, except for the last data point for each system, which is obtained using the $4880-\AA$ line at nearly $180^{\circ}$, to obtain larger scattering wave vectors and hence larger frequencies. The velocity $v$ for each system increases dramatically, by as much as $5 \%$, when the frequency increases from 5 to $8 \mathrm{GHz}$, corresponding to scattering angles varying from $90^{\circ}$ to $175^{\circ}$. This additional frequency dispersion in the velocity is observed in all AOT micelle and microemulsion systems, regardless of the size of the droplets or the solvent used. These results imply a further relaxation process, which results in a further increase in the stiffness of the mixture at higher frequency.

One possible origin for this behavior is the exchange of surfactant monomers between the micelles or microemulsions and the surrounding solvent oil. However, the characteristic frequency of this effect is generally believed to be much lower than the frequencies observed here [34]. Furthermore, the exchange frequency is considerably different for micelles and microemulsions, while the relaxation frequency observed here is essentially constant for all systems. Thus, we reject the surfactant monomer exchange as the origin of this high-frequency relaxation.

Instead, we attribute this behavior as arising from additional relaxation due to the surfactant tails, since it is independent of the solvent and the droplet size and since the time scale is consistent with the frequencies associated with the motion of the surfactant tails. Thus, there is an additional characteristic time $\tau_{t}$ corresponding to the motion of the tails. The inverse of this relaxation time is slightly above $5 \mathrm{GHz}$. When the period of the sound wave is shorter than $\tau_{t}$, there is not enough time for this tail motion to relax, and the system appears stiffer, thereby increasing the velocity of sound. This additional internal molecular degree of freedom may also contribute to

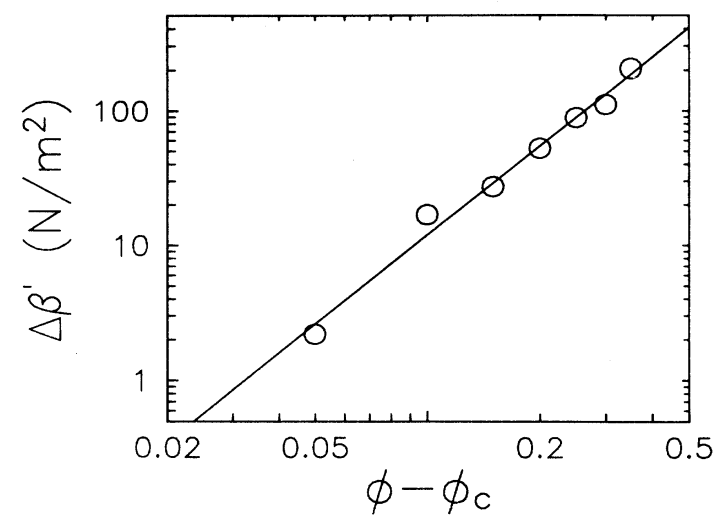

FIG. 9. Additional elastic modulus due to the relaxation of the tails of AOT surfactant molecules in the system as a function of volume fraction, demonstrating a further enhancement of the dynamic rigidity. The solid lines are fits to $\left(\phi-\phi_{c}\right)^{\tau}$ with $\tau \simeq 2.5$ and $\phi_{c}=0.16$, the same values that describe the behavior of the dynamic rigidity percolation observed at lower frequencies.

the stiffness of the extended networks formed by the droplets. To examine this possibility, in Fig. 9 we plot the increase in the elastic modulus from $1 \mathrm{GHz}$, which is the plateau of the relaxation due to the networks, to 8 $\mathrm{GHz}$, which is the highest frequency measured, as a function of $\phi-\phi_{c}$, for the micelles in decane. Unlike the previous dispersion observed at lower frequencies, we are unable to reach the asymptotic value of $v$ at the highest frequencies. We again find that the data exhibit a powerlaw scaling. As before, the data are well described by $\Delta \beta^{\prime} \sim\left(\phi-\phi_{c}\right)^{\tau}$, with $\phi_{c} \sim 0.16$ and $\tau \sim 2.5$, in surprising accord with the previous values. This implies that the additional rigidity contributed by the "frozen" surfactant at high frequency also contributes to the percolating networks. At higher $\phi$, there are more AOT molecules in the system to contribute to the enhancement of the elastic modulus, making it a function of droplet volume fraction with a behavior similar to that of the droplet networks themselves.

\section{CONCLUSION}

We have presented the results of a systematic study of sound propagation in AOT micelles and microemulsions. These systems are comprised of the surfactant, sodium di-2-ethylhexylsulfosuccinate (AOT), in a solvent of a linear chain hydrocarbon. When no water is added, the surfactant aggregates into inverted micelles each containing 22 AOT molecules with a radius of $15 \AA$. Upon addition of water, microemulsion droplets are formed with a water core and surfactant shell. In all cases, the droplet diameter remains unchanged as the droplet concentration is varied, allowing us to measure the dependence of the $v$, the sound velocity, on the volume fraction $\phi$ of micelles or microemulsions. For all cases, we have used ultrasonic and Brillouin-scattering measurements to determine the frequency dispersion in the sound velocity over three and a half decades. 
A consistent physical picture is presented to account for all of our observations. Because of the close-packed, polar head groups of the surfactant, the droplets are more rigid than the solvent phase. Thus the sound velocity always increases with $\phi$. In addition, there is a weak, short-range, attractive interaction between the overlapping tails of the surfactant molecules. As a result, the droplets can form short-lived, extended networks, and these networks can support shear, provided they span the whole system. In that case, the additional shear modulus increases the rigidity of the system, further increasing the sound velocity. This accounts for the additional dispersion in the velocity observed at high frequencies and high volume fractions. The magnitude of the attractive interaction increases with the chain length of the solvent molecules. Thus the onset frequency for the additional dispersion in $v$ at high $\phi$ due to the shear modulus of the networks decreases as the solvent is changed from hexane to decane to hexadecane. In addition, the magnitude of the attractive interaction increases upon further addition of water, since the area of overlap is greater with increasing droplet radius for the microemulsions. Thus, again the onset frequency for the contribution of the shear modulus of the networks decreases as the droplet radius increases upon addition of water. However, the water cores of the microemulsion droplets decrease the inherent rigidity of droplets themselves, and this effect competes with increasing rigidity of the surfactant shells. Thus, as the droplet radius increases further, the shear modulus of the microemulsion phase is ultimately no longer observed.

Essential to the interpretation of our data in the context of this physical picture is the theoretical description of the $\phi$ dependence of $v$ using an effective-medium model. At low frequencies, where the extended networks are short lived and do not contribute a shear modulus, the droplets act as independent particles. At higher frequencies, we treat the droplets as a continuous, connected phase, and include the shear modulus of this phase in the calculation of the $\phi$ dependence of the longitudinal elastic modulus of the composite medium. To account for the frequency dispersion of $v$, we allow the parameters which characterize the elastic properties of the droplet phase to be frequency dependent, and fit the $\phi$ dependence of $v$ for each frequency. This allows us to determine the magni- tude of the contribution of the shear modulus of the droplet phase.

Since the extended networks of droplets can only support shear if they span the system, they can only contribute an additional shear rigidity above a critical volume fraction $\phi_{c}$. Furthermore, the magnitude of this shear rigidity exhibits a scaling behavior in volume fraction above $\phi_{c}$. Thus the shear modulus of the droplets exhibits dynamic rigidity percolation. We experimentally demonstrate this percolation behavior by extracting the shear modulus from the additional increase in $v$ with frequency. We show that both the micelles and microemulsions exhibit nearly identical dynamic rigidity percolation behavior.

Finally, we also show that the full Rayleigh-Brillouin spectra obtained can be described in terms of the formalism developed by Mountain [12]. This formalism includes the contribution of an internal relaxation of the medium, which results in additional scattering intensity between the Rayleigh peak and the Brillouin doublet. We attribute this internal relaxation to that of the extended networks. Excellent fits of the spectra are obtained, and the values for the relaxation time of the networks are in good accord with the values determined directly from the measured dispersion of the sound velocity. Thus a self-consistent picture is obtained. We also discuss an additional dispersion in $v$ that is observed at very high frequencies, using Brillouin scattering at large scattering angles. This dispersion is observed for all systems at a similar frequency, and is therefore attributed to the relaxation of the tails of the surfactant molecules themselves. This additional dispersion also contributes to the rigidity of the droplet networks, and thus also exhibits a percolation scaling with volume fraction.

In conclusion, these results demonstrate the utility of using sound propagation to probe the behavior and dynamics of micelle and microemulsion systems. They also demonstrate the richness of the behavior exhibited by these systems.

\section{ACKNOWLEDGMENTS}

The authors are grateful to M. J. Higgins and J. Sung for their technical support, and to Dr. S. Bhattacharya for many helpful discussions.
[1] B. J. Berne and R. Pecora, Dynamic Light Scattering (Wiley, New York, 1976).

[2] E. F. Carome, W. H. Nichols, C. R. Kunsitis-Swyt, and S. P. Singal, J. Chem. Phys. 49, 1013 (1968).

[3] H. Z. Cummins and R W. Gammon, J. Chem. Phys. 44, 2785 (1966)

[4] W. H. Nichols, C. R. Kunsitis-Swyt, and S. P. Singal, J. Chem. Phys. 51, 5659 (1969).

[5] P. A. Fleury and J. P. Boon, Phys. Rev. 186, 244 (1969).

[6] L. Ye, D. A. Weitz, P. Sheng, S. Bhattacharya, J. H. Huang, and M. J. Higgins, Phys. Rev. Lett. 63, 263 (1989).

[7] L. Ye, D. A. Weitz, P. Sheng, and J. H. Huang, in Macro- molecular Fluids, edited by C. R. Safinya, S. Safran, and P. Pincus, MRS Symposia Proceedings No. 177 (Materials Research Society, Pittsburgh, 1990), p.111.

[8] M. Kotlarchyk and J. S. Huang, J. Phys. Chem. 89, 1985 (1985).

[9] M. Kotlarchyk, S. H. Chen, and J. S. Huang, J. Phys. Chem. 86, 3273 (1982).

[10] J. S. Huang, J. Chem. Phys. 82, 480 (1985).

[11] P. Sheng, in Homogenization and Effective Modulii of Materials and Media, edited by J. L. Ericksen, D. Kinderleher, R. Kohn, and J. L. Lions (Springer-Verlag, New York, 1983), p. 196. 
[12] R. D. Mountain, C.R. Acad. Sci. 1, 5 (1970).

[13] Surfactants in Solution, edited by K. Mittal and B. Lindman (Plenum, New York, 1984).

[14] T. Assin, F. Larche and P. Delord, J. Colloid Interface Sci. 89, 35 (1982).

[15] J. S. Huang, S. A. Safran, M. W. Kim, G. S. Grest, M. Kotlarchyk, and N. Quirke, Phys. Rev. Lett. 53, 592 (1984).

[16] G. T. Kuster and M. N. Toksoz, Geophysics 39, 587 (1974).

[17] Z. Hashin, J. Appl. Mech. 29, 143 (1962).

[18] Handbook of Chemistry and Physics, edited by R. C. Weast (Chemical Rubber, Cleveland, 1986), Vol. 66.

[19] R. Zallen, The Physics of Amorphous Solids (Wiley, New York, 1983), p. 49.

[20] D. Stauffer, Introduction to Percolation Theory (Taylor and Francis, London, 1985), p. 52.

[21] P. G. de Gennes, J. Phys. Lett. 37, L1 (1976).

[22] S. Alexander, J. Phys. (Paris) 45, 1939 (1984).

[23] S. Feng, P. Sen, B. I. Halperin, and C.J. Lobb, Phys. Rev. B 30, 5386 (1984).

[24] Y. Kantor and I. Webman, Phys. Rev. Lett. 52, 1891
(1984).

[25] D. Deptuck, J. P. Harrison, and P. Zawadzki, Phys. Rev. Lett. 54, 913 (1985).

[26] S. A. Safran, I. Webman, and G. S. Grest, Phys. Rev. A 32, 506 (1985).

[27] B. Lagourette, J. Peyrelasse, C. Boned, and M. Clausse, Nature 281, 60 (1981).

[28] M. J. Lagues, J. Phys. (Paris) Lett. 40, L339 (1979); S. Bhattacharya, J. P. Stokes, M. W. Kim, and J. H. Huang, Phys. Rev. Lett. 55, 1884 (1966).

[29] R. D. Mountain, J. Chem. Phys. 44, 832 (1966).

[30] W. H. Nichols and E. F. Carome, J. Chem. Phys. 49, 1000 (1968).

[31] P. J. Carroll and G. D. Patterson, J. Chem. Phys. 81, 1666 (1984).

[32] G. D. Patterson and C. P. Lindsey, J. Appl. Phys. 49, 5039 (1978).

[33] G. D. Patterson, Methods of Experimental Plysics (Academic, New York 1980), p. 170.

[34] S. Harada and Z. A. Schelly, J. Phys. Chem. 86, 2098 (1982). 